\title{
EMBARAZO ECTÓPICO NO COMPLICADO: MANEJO MÉDICO CON METOTREXATE EN EL INSTITUTO NACIONAL MATERNO PERINATAL. ENERO 2009 - DICIEMBRE 2010
}

\author{
Christian Arturo Zambrano Gamboa1, Mirasol Pavel Ticona Huamaní2, \\ Agustín Fernando Murga López ${ }^{3}$, Luis Carpio Guzmán ${ }^{4}$
}

\begin{abstract}
RESUMEN
Objetivo. Determinar las características del manejo médico con metotrexate en el embarazo ectópico no complicado en el Instituto Nacional Materno Perinatal de Lima. Materiales y métodos. Estudio tipo Investigación observacional analítica. Diseño: Observacional, descriptivo, retrospectivo, de casos. La muestra estuvo comprendida por 30 historias clínicas, de pacientes con diagnóstico de embarazo ectópico que recibieron metotrexate en el periodo enero 2009 - diciembre 2010. Los instrumentos empleados estuvieron conformados por una ficha de recolección de datos elaborada para los fines de estudio. Resultados. La localización más frecuente fue la tubárica derecha en el $56,7 \%$ de los casos, el esquema más usado fue el de la dosis única en el $86,6 \%$ de los casos, el tamaño más frecuente fue el de 20 a $35 \mathrm{~mm}$ en el $60 \%$ de los casos, el $70 \%$ de los casos negativizaron la B-hCG (fracción B de la gonadotropina coriónica humana), el 30\% requirió cirugía, la reacción adversa más frecuente al metotrexate fue las nauseas (10\% de los casos).Conclusiones. El tratamiento médico con metotrexate es una alternativa para pacientes con embarazo ectópico no complicado, presentando una alta frecuencia de negativización de B-hCG (70\%). No hubo diferencias estadísticamente significativas en cuanto a los valores de la B-hCG al momento de la decisión quirúrgica. El tipo de esquema más usado fue el de una dosis y la vía de administración fue la vía intramuscular en todas las pacientes.
\end{abstract}

Palabras clave: Embarazo ectópico; Metotrexate.

\section{NO COMPLICATED ECTOPIC PREGNANCY: MEDICAL MANAGEMENT WITH METHOTREXATE IN THE MATERNAL PERINATAL NATIONAL INSTITUTE. JANUARY 2009 - DECEMBER 2010}

\begin{abstract}
Objective. To determine the characteristics of medical management with methotrexate in unruptured ectopic pregnancy in Instituto Nacional Materno Perinatal in Lima. Material and methods. A descriptive study, retrospective, realized between January 2009 and December 2010. These were 30 patients diagnosed with unruptured ectopic pregnancy who received methotrexate in the study period. The instrument used was a data collection form developed for study purposes. Results. The most frequent was tubal right at the $56,7 \%$ of cases, the most widely used protocol was single-dose in $86,6 \%$ of cases, the most common size was 20 to $35 \mathrm{~mm}$ in $60 \%$ of cases, $70 \%$ of cases became negative B-hCG (fraction B of human chorionic gonadotropin), $30 \%$ required surgery, the most frequent adverse reaction to methotrexate was nausea ( $10 \%$ of cases).Conclusions. Medical management with methotrexate is an alternative for patients with unruptured ectopic pregnancy, became negative B-hCG (70\%). There was no statistically significant differences in the values of the B-hCG at the time of surgical decision. The most widely used protocol was single-dose and route of administration was intramuscularly in all patients.
\end{abstract}

Key words: Ectopic pregnancy; Methotrexate.

\section{INTRODUCCIÓN}

El embarazo ectópico es la implantación del blastocisto en un sitio diferente al endometrio de la cavidad uterina normal ${ }^{1}$, y sigue siendo la principal causa de muerte en el primer trimestre relacionada con el embarazo entre las mujeres en los Estados Unidos ${ }^{2}$.
Los embarazos ectópicos representan aproximadamente el $2 \%$ de todos los embarazos según la mayoría de literatura mundial ${ }^{3,4}$.

De acuerdo a cifras nacionales ${ }^{5}$, en el Instituto Materno Perinatal, el año 2001 la incidencia de embarazo ectópico fue de 3,3 por 1000 gestaciones, cifra compartida por

Médico Gineco Obstetra, Asistente del Departamento de Cuidados Críticos, Instituto Nacional Materno Perinatal

Médico Gineco Obstetra, Ex Residente del Instituto Nacional Materno Perinatal

Médico Gineco Obstetra, jefe del Departamento de Ginecología, Instituto Nacional Materno Perinatal

Médico Gineco Obstetra Asistente del Departamento de Ginecología, Instituto Nacional Materno Perinatal

Recibido: 10-10-11 Aprobado: 23-07-12 
otros estudios nacionales; pero menor a lo reportado en Estados Unidos y países occidentales.

En el momento actual se acepta que en todo el mundo ha ocurrido un incremento en la incidencia de embarazos ectópicos, atribuible probablemente a la mayor frecuencia de inflamaciones pélvicas, dispositivos intrauterinos, técnicas de reproducción asistida, etc., lo que constituye un serio problema para la Obstetricia moderna.

La importancia del embarazo ectópico radica en que es una condición que puede comprometer seriamente la salud de la mujer, pues todavía causa muertes maternas en nuestro país y en todo el mundo, y tiene impacto en la fertilidad futura.

En el pasado el tratamiento del embarazo ectópico era siempre quirúrgico. Con el advenimiento de nuevas técnicas diagnosticas y de procederes terapéuticos, su conducta ha cambiado mucho y se ha favorecido la conservación de las trompas.

En Japón, en el año 1982 se realizaron los primeros tratamientos, por los cuales se recomendó el uso de metotrexate para el embarazo intersticial ${ }^{6}$.

El uso del metotrexate para el embarazo ectópico fue propuesto después de la observación de que la replicación activa del trofoblasto en la enfermedad trofoblástica gestacional era tratada con éxito con metotrexate ${ }^{7,8}$

El metotrexato, agente quimioterápico, es un antagonista del ácido fólico, inhibidor de la reductasa del ácido fólico que inhibe la síntesis espontánea de purinas y pirimidinas e interfiere así la síntesis de DNA y la multiplicación celular. Aunque no se ha demostrado el efecto fisiopatológico específico en del metotrexate en el embarazo ectópico, pero se cree que induce aborto tubario y/o reabsorción del mismo ${ }^{9}$.

Después de ser usado exitosamente por más de 20 años en el manejo médico del embarazo ectópico, el metotrexate ha sido ampliamente aceptado y actualmente se le considera el tratamiento médico de elección para pacientes hemodinámicamente estables ${ }^{10}$.

De esta forma, el embarazo ectópico ha pasado a ser una enfermedad con posibilidad de tratamiento médico, lo que implica conocer adecuadamente las indicaciones y características de los fármacos utilizados, a fin de lograr la máxima efectividad con esta alternativa terapéutica, que evita el riesgo que conlleva una cirugía.

El diagnóstico precoz puede reducir la mortalidad y la morbilidad asociada con el embarazo ectópico. Las dos pruebas diagnósticas más importantes son la ecografía transvaginal y el dosaje del nivel sérico de gonadotropina coriónica humana. La sensibilidad y especificidad de la combinación de estas pruebas se ha informado que van desde 95 a $100 \%{ }^{11}$.
Después de que se realiza el diagnóstico, varios factores influyen en la decisión de tratar un embarazo ectópico médica o quirúrgicamente. Si el paciente se encuentra inestable, el tratamiento quirúrgico inmediato mediante laparotomía o laparoscopia es necesario ${ }^{12}$.

Aquellos pacientes hemodinámicamente estables son candidatos para recibir tratamiento médico con metotrexate.

Los criterios de inclusión y exclusión para la administración de metotrexate han sido recomendados por el Colegio Americano de Obstetricia y Ginecología ${ }^{7}$, los cuales incluyen, indicaciones absolutas:

Paciente hemodinámicamente estable, sin sangrado activo o signos de hemoperitoneo.

Paciente con deseo de fertilidad.

Paciente en condiciones de volver para el seguimiento.

Paciente en el cual la anestesia general supone un riesgo.

Paciente quien no tiene contraindicación para metotrexate

Indicaciones relativas:

Masa no rota $<3,5 \mathrm{~cm}$ en su mayor dimensión.

No actividad cardiaca fetal.

Que la $\beta$-hCG no exceda un valor máximo predeterminado

Contraindicaciones absolutas:

Lactancia materna.

Inmunodeficiencia.

Valores anormales de creatinina ( $>1,3 \mathrm{mg} / \mathrm{dL})$, aspartato aminotransferasa (dos veces el valor normal).

Alcoholismo o enfermedad hepática.

Discrasias sanguíneas preexistentes.

Úlcera péptica.

Enfermedad pulmonar activa.

Sensibilidad conocida al metotrexate.

Contraindicaciones relativas:

Saco gestacional $>3,5 \mathrm{~cm}$.

Actividad cardiaca fetal.

Desde su introducción, diversos protocolos para el uso de metotrexato en el tratamiento del embarazo ectópico han sido propuestos, siendo el protocolo de dosis única de metotrexato presentado por Stovall y colaboradores el más comúnmente utilizado ${ }^{7,13}$.

La tasa de éxito reportado tanto para el esquema de dosis única como para el esquema de dosis múltiple es aproximadamente $90 \%{ }^{3}$.

El esquema de tratamiento con metotrexato sistémico en dosis única resulta en ahorros significativos en costos directos e indirectos en comparación con el tratamiento quirúrgico convencional como la cirugía laparoscópica. Según Alexander et al ${ }^{14}$ el tratamiento con metotrexate resultarì 2 a 6 veces más costo-efectivo en la resolución del embarazo ectópico no complicadoen comparación 
con el tratamiento quirúrgico. Estos ahorros en costos se deben tanto al uso reducido de material como a la estancia hospitalaria ${ }^{13}$.

Hasta la fecha, la serie más grande reportada de pacientes quienes fueron tratados con protocolo de dosis única de metotrexate describió pacientes que fueron incluidos en un estudio clínico en Memphis, Tennessee ${ }^{15}$. Sin embargo, sólo hay información limitada sobre la experiencia real de esquema de dosis única de metotrexate en el ámbito clínico fuera del cumplimiento estricto de un protocolo de investigación ${ }^{16}$.

Un aspecto importante observado por Cruz $^{5}$ en el Instituto Nacional Materno Perinatal es que, siendo en la laparoscopia el método quirúrgico estándar para el tratamiento del embarazo ectópico complicado; no se practica con regularidad en la institución. Asimismo, el 2001, no se encontró ningún caso con tratamiento médico.

El Instituto Nacional Materno Perinatal es una institución de referencia nacional de patología obstétrica. En los últimos años se ha evidenciado un incremento de la incidencia de embarazo ectópico y del manejo médico con metotrexate de esta patología. Sin embargo, en la práctica se ha observado, que en nuestro país, se considera muy poco esta alternativa terapéutica, siendo la institución una de las pioneras en incorporarla a la práctica clínica.

Pues como podemos ver en la revisión de la literatura son pocos los estudios realizados en nuestro medio con respecto al tema, es por ello que consideramos la importancia de la realización del presente trabajo de investigación.

El objetivo del presente estudio fue determinar las características y el resultado que se obtiene con el manejo médico con metotrexate en el embarazo ectópico no complicado en el Instituto Nacional Materno Perinatal de Lima - Perú durante el período comprendido entre el 01 de Enero de 2009 y el 31 de Diciembre de 2010.

\section{MATERIALES Y MÉTODOS}

Tipo de estudio: Investigación observacional analítica.

Diseño de investigación: Observacional descriptivo, retrospectivo y transversal.

Población de estudio: El universo de estudio incluyó aquellos pacientes con diagnóstico de embarazo ectópico no complicado que fueron atendidas en el Instituto Nacional Materno Perinatal durante el periodo entre 01 de enero del 2009 y 31 de diciembre de 2010; en nuestro estudio contamos con 30 casos de embarazo ectópico tratadas con metrotexate.

Tamaño de la muestra: Se trabajó con el total de pacientes con diagnóstico de embarazo ectópico no complicado que recibieron tratamiento médico con metotrexate en dosis de $50 \mathrm{mg} / \mathrm{m} 2$ de superficie corporal en administrada por vía intramuscular.

Los instrumentos empleados estuvieron conformados por una ficha de recolección de datos elaborada para los fines de estudio.

\section{Criterios de inclusión:}

Paciente hemodinámicamente estable que recibió tratamiento con metotrexate por el diagnóstico de embarazo ectópico no complicado en el Servicio de Ginecología del Instituto Nacional Materno Perinatal.

Se requirió dosaje de $\beta$-hCG en sangre al inicio del tratamiento y durante el seguimiento.

Tamaño del embarazo ectópico o masa anexial no mayor de $3,5 \mathrm{~cm}$.

Ausencia de líquido libre en la cavidad pélvica al examen ultrasonográfico.

Ausencia de embrión con actividad cardíaca.

Ausencia de antecedentes de enfermedades renales, hematológicas o hepáticas.

Hemograma, urea, creatinina y transaminasas normales antes del inicio del tratamiento.

Criterios de exclusión:

Enfermedad hepática conocida o documentada en la historia clínica.

Antecedente documentado de hipersensibilidad al metotrexate.

Historia clínica no accesible y/o con datos incompletos. Analisis estadístico.

Los datos obtenidos durante la investigación, por medio de la ficha de recolección de datos, se ordenaron y procesaron valiéndonos de los programa SPSS 18,0. Epi info y Minitab. Se observaron y analizaron los resultados y la posible aparición de relaciones entre ellos utilizando el método de Chi cuadrado $\left(\mathrm{X}^{2}\right)$ y prueba exacta de Fisher, en caso de variables cualitativas, y en los casos de las variables cuantitativas se aplicó el Test de Student. Para la elaboración de los gráficos de frecuencias se utilizó el programa Crystal Report. Para la medición de la fuerza de asociación se utilizó el OR (Odds Ratio) con un intervalo de confianza del $95 \%$.

\section{RESULTADOS}

En el periodo de estudio, de un total de 35241 nacidos vivos hubieron 121 embarazos ectópicos, que representa una prevalencia del $0,34 \%$. Se revisaron 30 historias clínicas, de pacientes hospitalizadas con diagnostico de embarazo 
ectópico no complicado en el servicio de Ginecobstetricia en el Instituto Nacional Materno Perinatal (Tabla 1).

En cuanto a las variables cuantitativas encontramos que respecto a la edad, la media fue de 31,4 años con una desviación estándar de 6,3 años; siendo la mínima edad de 17 años y la máxima de 43 años; no existiendo diferencias significativas en cuanto al esquema de Metotrexate usado en relación a la necesidad de cirugía.

En relación a los antecedentes obstétricos, encontramos que la media fue de 2,7 gestaciones, con una desviación

Tabla 1. Características generales de las pacientes con embarazo ectópico no complicado tratadas con metotrexate en el Instituto Nacional Materno Perinatal. Enero 2009 - diciembre 2010.

\begin{tabular}{|c|c|c|c|}
\hline \multicolumn{2}{|c|}{ Características Generales } & \multirow{2}{*}{$\frac{N}{5}$} & \multirow{2}{*}{$\begin{array}{c}\% \\
16,7 \%\end{array}$} \\
\hline Antecedente & Si & & \\
\hline ectopico & No & 25 & $83,3 \%$ \\
\hline \multirow{5}{*}{ Localización } & Cervical & 2 & $6,7 \%$ \\
\hline & Tubarico derecho & 17 & $56,7 \%$ \\
\hline & Tubarico izquierdo & 7 & $23,3 \%$ \\
\hline & Cornual derecho & 3 & $10,0 \%$ \\
\hline & Cornual izquierdo & 1 & $3,3 \%$ \\
\hline \multirow{6}{*}{ Edad gestacional } & 8 semanas & 8 & $26,7 \%$ \\
\hline & 6 semanas & 9 & $30,0 \%$ \\
\hline & 7 semanas & 8 & $26,7 \%$ \\
\hline & 9 semanas & 3 & $10,0 \%$ \\
\hline & 12 semanas & 1 & $3,3 \%$ \\
\hline & 10 semanas & 1 & $3,3 \%$ \\
\hline \multirow{3}{*}{$\begin{array}{l}\text { Esquema } \\
\text { metotrexate }\end{array}$} & Dosis única & 26 & $86,7 \%$ \\
\hline & Dos dosis & 3 & $10,0 \%$ \\
\hline & $\begin{array}{l}\text { Una dosis } \\
\text { con segunda dosis }\end{array}$ & 1 & $3,3 \%$ \\
\hline \multirow{4}{*}{$\begin{array}{l}\text { Tamaño masa } \\
\text { anexial }\end{array}$} & $20-35$ & 18 & $60,0 \%$ \\
\hline & $<20$ & 2 & $6,7 \%$ \\
\hline & $>35$ & 6 & $20,0 \%$ \\
\hline & $30-35$ & 4 & $13,3 \%$ \\
\hline \multirow{2}{*}{$\begin{array}{l}\text { Actividad } \\
\text { cardiáca embrión }\end{array}$} & $\mathrm{Si}$ & 2 & $6,7 \%$ \\
\hline & No & 28 & $93,3 \%$ \\
\hline \multirow{3}{*}{$\begin{array}{l}\text { Liquido en } \\
\text { cavidad }\end{array}$} & Escaso & 7 & $23,3 \%$ \\
\hline & No & 20 & $66,7 \%$ \\
\hline & Regular cantidad & 3 & $10,0 \%$ \\
\hline \multirow{2}{*}{ Negativizacion } & $\mathrm{Si}$ & 21 & $70,0 \%$ \\
\hline & No & 9 & $30,0 \%$ \\
\hline \multirow{2}{*}{ Cirugía } & $\mathrm{Si}$ & 9 & $30,0 \%$ \\
\hline & No & 21 & $70,0 \%$ \\
\hline \multirow{3}{*}{$\begin{array}{l}\text { Motivo } \\
\text { de cirugía }\end{array}$} & No & 21 & $70,0 \%$ \\
\hline & Incremento de b-hcg & 4 & $13,3 \%$ \\
\hline & $\begin{array}{l}\text { Aumento del dolor } \\
\text { abdominal }\end{array}$ & 5 & $16,7 \%$ \\
\hline \multirow{2}{*}{$\begin{array}{l}\text { Reacción } \\
\text { adversas al } \\
\text { tratamiento }\end{array}$} & No & 24 & $80,0 \%$ \\
\hline & $\mathrm{Si}$ & 6 & $20,0 \%$ \\
\hline \multirow{4}{*}{ Tipo reacción } & No & 24 & $80,0 \%$ \\
\hline & Naúseas & 3 & $10,0 \%$ \\
\hline & Dolor abdominal & 2 & $6,7 \%$ \\
\hline & $\begin{array}{l}\text { Náuseas, elevación de } \\
\text { enzimas hepáticas }\end{array}$ & 1 & $3,3 \%$ \\
\hline \multicolumn{2}{|l|}{ Total } & 30 & $100,0 \%$ \\
\hline
\end{tabular}

estándar de 1,2 gestas; siendo la mínima de una gestas y la máxima de 6 gestas; y en relación al número de abortos encontramos que la media fue de 0,76 abortos, con una desviación estándar de 0,77 abortos; no existiendo diferencias significativas en cuanto al esquema de Metotrexate usado en relación a la necesidad de cirugía.

En cuanto a la B-hCG al inicio encontramos que la media fue de 2134 UI, con una desviación estándar de 1411; siendo la mínima de 224 y la máxima de 6810Ul; no existiendo diferencias significativas en cuanto al esquema de Metotrexate usado en relación a la necesidad de cirugía.

En cuanto al tiempo de negativización de la B-hCG encontramos que la media del tiempo de negativización fue de 4,7 semanas, con una desviación estándar de 3,8 semanas; siendo la mínima antes de la primera semana y la máxima de 12 semanas.

En relación a las características generales de las pacientes encontramos los siguientes resultados: antecedente de embarazo ectópico hubo una frecuencia de antecedente positivo en el $16,7 \%$ de los casos. La localización del embarazo ectópico hubo una mayor frecuencia de localización tubárica derecha en el $56,7 \%$ de los casos.

En relación a la edad gestacional encontramos una mayor frecuencia de gestación de 6 semanas en el $30 \%$ de los casos.

El esquema de metotrexate mas usado fue el de dosis única en el $86,7 \%$ de los casos.

En cuanto al tamaño de la masa anexial encontramos una mayor frecuencia de tamaño entre 20 y $35 \mathrm{~mm}$ en el $60 \%$ de los casos.

En relación a la actividad cardiaca del embrión encontramos que hubo actividad en el $6,7 \%$ de los casos.

En cuanto al líquido en cavidad pélvica encontramos que hubo líquido escaso en el $23,3 \%$ de los casos.

En cuanto a la negativización; encontramos que el $70 \%$ de los casos negativizó.

El $30 \%$ de los casos requirió cirugía, siendo el motivo más frecuente el aumento del dolor abdominal.

La reacción adversa más frecuente fueron las nauseas en el $10 \%$ de los casos; el $80 \%$ de los casos no tuvo reacción adversa.

En la tabla 2 se muestra en relación a las medias de la $\beta$-HCG y la necesidad de cirugía encontramos que las pacientes que fueron operadas tuvieron una media de $2269 \mathrm{UI}, \mathrm{y}$ en las que no fueron operadas la media fue de $2077 \mathrm{UI}$, no siendo esta diferencia estadísticamente significativa $(P>0,05)$. 
Tabla 2. Medias de la $\beta-H C G$ según necesidad de cirugía en las pacientes con embarazo ectópico no complicado en el Instituto Nacional Materno Perinatal. Enero 2009 - diciembre 2010.

\begin{tabular}{llccccccc}
\hline Cirugía & \multicolumn{1}{c}{ Motivo de cirugía } & media & $\mathbf{n}$ & Desv típ & mínimo & máximo & $\%$ del total de N & $\mathbf{p}$ \\
\hline \multirow{4}{*}{ Si } & Incremento de $\beta$-HCG & 2096,0000 & 4 & 1188,05163 & 780,00 & 3664,00 & $13,3 \%$ \\
& Aumento del dolor & 2408,6000 & 5 & 1505,60762 & 611,00 & 4370,00 & $16,7 \%$ \\
& abdominal & 2269,6667 & 9 & 1299,95048 & 611,00 & 4370,00 & $30,0 \%$ \\
& Total & 2077,2381 & 21 & 1483,26737 & 224,00 & 6810,00 & $70,0 \%$ & \\
\multirow{2}{*}{ No } & No & 2077,2381 & 21 & 1483,26737 & 224,00 & 6810.00 & $70,0 \%$ & $P>0,05$ \\
& Total & 2077,2381 & 21 & 1483,26737 & 224,00 & 6810,00 & $70,0 \%$ \\
& No & 2096,0000 & 4 & 1188,05163 & 780,00 & 3664,00 & $13,3 \%$ \\
\multirow{2}{*}{ Total } & Incremento de $\beta$-HCG & Aumento del dolor & 2408,6000 & 5 & 1505,60762 & 611,00 & 4370,00 & $16,7 \%$ \\
& abdominal & 2134,9667 & 30 & 1411,20996 & 224,00 & 6810,00 & $100,0 \%$ \\
& Total & & & & & & \\
\hline
\end{tabular}

En la Tabla 3 se muestra en relación a las medias de la $\beta$-HCG según cirugía en relación al tamaño anexial; en las pacientes con embarazo ectópico encontramos una mayor frecuencia de tamaño entre 20 y $35 \mathrm{~mm}$ en un $16,7 \%$ de los casos; siendo la media de la $\beta$-HCG de $2718 \mathrm{UI}$, y en las que no fueron operadas hubo una mayor frecuencia de tamaños entre los 20 y $35 \mathrm{~mm}$; siendo la media de la $\beta$-HCG de $1743 \mathrm{UI}$, no siendo esta diferencia estadísticamente significativa.

En cuanto a las medias de la edad en relación a la necesidad de cirugía encontramos que la media de las pacientes que si fueron operadas fue de $30,77 \pm 6,9$ años y en las pacientes que no fueron operadas la media de la edad fue de $31,73 \pm 6,2$ años, no siendo esta diferencia estadísticamente significativa( $P>0,05)$.

En cuanto a la localizacion del embarazo ectópico en relación a la necesidad de cirugía encontramos que en las pacientes que si requirieron cirugía hubo una mayor frecuencia de localización tubárica derecha en el 66,7\%; y en las pacientes que no requirieron cirugía también hubo una mayor frecuencia de localización tubárica derecha en $52,4 \%$, no siendo esta diferencia estadísticamente significativa $(p>0,05)$.
En cuanto al área bajo la curva para las variables estudiadas; encontramos que el antecedente de abortos, el tiempo de negativización y la persistencia de dolor abdominal fueron las variables que predicen significativamente la conducta quirúrgica $(p<0,05)$.

\section{DISCUSIÓN}

Desde la publicación de Tanaka6 en 1982, se han publicado numerosos artículos sobre el uso de metotrexate parenteral ${ }^{17,18}$; así como diversos algoritmos y protocolos para el diagnóstico y tratamiento médico, los mismos que incluyen dosis única, dos dosis y múltiples dosis ${ }^{19,20}$. De ellos, el protocolo de dosis única presentado por Stovall et $\mathrm{al}^{7}$ es el más utilizado; dato que coincide con nuestro estudio donde encontramos que en el 86,6 \% de los casos se usaron dosis única por vía intramuscular.

Así mismo, el Colegio Real de Obstetras y Ginecólogos del Reino Unido recomienda el esquema de dosis única de metotrexate para el tratamiento médico del embarazo ectópico.

Bueno et al ${ }^{21}$, en un estudio con 21 casos de embarazo ectópico no complicados que fueron tratados con

Tabla 3. Medias de la $\beta$-hCG según cirugía en relación al tamaño anexial en las pacientes con embarazo ectópico no complicado en el Instituto Nacional Materno Perinatal periodo enero 2009 a diciembre 2010.

\begin{tabular}{|c|c|c|c|c|c|c|c|}
\hline Cirugía & Tamaño masa anexial & Media & $\mathbf{N}$ & Desv. Típ. & Mínimo & Máximo & $\%$ del total de $n$ \\
\hline \multirow{5}{*}{$\mathrm{Si}$} & $20-35$ & 2718,2000 & 5 & 1399.00722 & 780,00 & 4370,00 & $16,7 \%$ \\
\hline & $<20$ & 3107,0000 & 1 & & 3107,00 & 3107,00 & $3,3 \%$ \\
\hline & $>35$ & 1251,5000 & 2 & 905.80379 & 611,00 & 1892,00 & $6,7 \%$ \\
\hline & $30-35$ & 1226,0000 & 1 & & 1226,00 & 1226,00 & $3,3 \%$ \\
\hline & Total & 2269,6667 & 9 & 1299.95048 & 611,00 & 4370,00 & $30,0 \%$ \\
\hline \multirow{5}{*}{ No } & $20-35$ & 1743,6923 & 13 & 817.71729 & 415,00 & 2850,00 & $43,3 \%$ \\
\hline & $<20$ & 2514,0000 & 1 & & 2514,00 & 2514,00 & $3,3 \%$ \\
\hline & $>35$ & 3879,0000 & 4 & 2238.40211 & 1370,00 & 6810,00 & $13,3 \%$ \\
\hline & $30-35$ & 974,6667 & 3 & 1156.92451 & 224,00 & 2307,00 & $10,0 \%$ \\
\hline & Total & 2077,2381 & 21 & 1483.26737 & 224,00 & 6810,00 & $70,0 \%$ \\
\hline \multirow{5}{*}{ Total } & $20-35$ & 2014,3889 & 18 & 1065.00954 & 415,00 & 4370,00 & $60,0 \%$ \\
\hline & $<20$ & 2810,5000 & 2 & 419.31432 & 2514,00 & 3107,00 & $6,7 \%$ \\
\hline & $>35$ & 3003,1667 & 6 & 2238.60764 & 611,00 & 6810,00 & $20,0 \%$ \\
\hline & $30-35$ & 1037,5000 & 4 & 952.94718 & 224,00 & 2307,00 & $13,3 \%$ \\
\hline & Total & 2134,9667 & 30 & 1411.20996 & 224,00 & 6810,00 & $100,0 \%$ \\
\hline
\end{tabular}


metotrexate y manejo expectante, no hubo correlación entre el nivel inicial de $\beta$-HCG con el tamaño de masa anexial, tiempo de negativización de $\beta$-HCG, necesidad de segunda dosis de metotrexate ni aparición de complicaciones del embarazo ectópico durante el tratamiento; estos datos también coinciden con nuestro estudio donde hallamos que no encontramos asociación entre el tamaño y la necesidad de cirugía. En dicho estudio tres casos requirieron resolución quirúrgica, determinando una tasa de éxito de tratamiento médico de $85,7 \%$. En nuestro estudio encontramos una tasa de éxito del tratamiento médico en un $70 \%$ de los casos.

Rojas et al ${ }^{22}$ realizaron un estudio en el Hospital Juárez de México, con el objetivo de analizar la eficacia e inocuidad del metotrexato administrado por vía intramuscular, en dosis única, en el tratamiento del embarazo ectópico no roto. Encontraron que la cuantificación basal de la la $\beta$-hCG fue, en promedio, de 14,760 mUI/L y la resolución del problema sucedió, en promedio, a los 45,6 4 19,85 días posteriores al tratamiento; dato que coincide con nuestro estudio donde la media del tiempo de negativización fue de 4,7 semanas con una desviación estándar de 3,8 semanas.

Aunque el diagnóstico precoz puede contribuir a una mayor incidencia, también ha contribuido a una disminución de la morbilidad, muertes y costos de tratamiento. El diagnóstico precoz y oportuno ha permitido que el embarazo ectópico sea susceptible de tratamiento médico, con tasas de éxito similares a los de la cirugía tradicional, la misma que se prefiere cuando hay rupturas de trompas o un alto potencial para la ruptura, hipotensión, anemia o embarazo ectópico, mayor de $3 \mathrm{~cm}$ de diámetro.

Huamán et al ${ }^{23}$ realizaron un estudio observacional prospectivo con 21 mujeres entre los años 1998 - 2005 con el objetivo de evaluar la respuesta al metotrexate en el tratamiento del embarazo ectópico tubárico; y concluyeron que el tratamiento con metotrexate, en casos seleccionados de embarazo ectópico, es una buena opción terapéutica, con 95,2\% de resultados satisfactorios.

En conclusión, el tratamiento médico con metotrexate es una alternativa para pacientes con embarazo ectópico no complicado, presentando una alta frecuencia de negativización de B-hCG (70\%). No hubo diferencias estadísticamente significativas en cuanto a los valores de la B-hCG al momento de la decisión quirúrgica.. El tipo de esquema más usado fue el de una dosis y la vía de administración utsada fue la intramuscular en todas las pacientes. La localización más frecuente fue la tubárica derecha y el tamaño mas frecuente fue de 20 a $25 \mathrm{~mm}$.

\section{REFERENCIAS BIBLIOGRÁFICAS}

1. Cotlar AM. Extrauterine pregnancy: a historical review. Curr Surg 2000; 57:484-92.

2. Mukul TS. Current management of Ectopic Pregnancy. N Am Obstet Gynecol Clin 2007 (34): 403-9.
3. Centers for Disease Control and Prevention. Ectopic pregnancyUnited States, 1990-1992. MMWR Morb Mortal Wkly Rep 1995; 44:46-8.

4. Grimes DA. The morbidity and mortality of pregnancy: still risky business. Am J Obstet Gynecol 1994; 170(5):1489-94ACOG Practice Bulletin No. 94: Medical management of ectopic pregnancy. Obstet Gynecol 2008; 111(6): 1479-85.

5. Cruz G. Incidencia y perfil obstétrico y quirúrgico de pacientes con embarazo ectópico en el Instituto Materno Perinatal, enero - diciembre 2001. Trabajo de Investigación. Especialista en Ginecología y Obstetricia. Universidad Nacional Mayor de San Marcos, Biblioteca de la Facultad de Medicina Humana Lima, 2002

6. Tanaka T, Hayashi H, Kutsuzawa T, Fujimoto S, Ichinoe K: Treatment of intertitial ectopic pregnancy with methtorexate: report of a successful case. Fertil Steril 1982; 37(6): 851-2.

7. ACOG Practice Bulletin No. 94: Medical management of ectopic pregnancy. Obstet Gynecol 2008; 111(6): 1479-85.

8. Sand PK, Stubblefield PA, Ory SJ. Methotrexate inhibition of normal trophoblasts in vitro. Am J Obstet Gynecol 1986; 155(2):324-9.

9. Menon S, Colins J, Barnhart KT. Establishing a human chorionic gonadotropin cutoff to guide methotrexate treatment of ectopic pregnancy: a systematic review. Fertil Steril 2007; 87 (3):481-4.

10. Royal College of Obstetricians and Gynecology. The management of tubal pregnancy. Guideline $N^{\circ} 21 ; 2004$.

11. Aleem FA, DeFazio M, Gintautas J. Endovaginal sonography for the early diagnosis of intrauterine and ectopic pregnancies. Hum Reprod 1990; 5(6):755-8.

12. Hajenius PJ, Mol BW, Bossuyt PM, et al. Interventions for tubal ectopic pregnancy. Cochrane Database Syst Rev. 2000; 2: CD000324.

13. Stovall TG, Ling FW, Gray LA. Single-dose methotrexate for treatment of ectopic pregnancy. Obstet Gynecol 1991; 77:754-7.

14. Alexander JM, Rouse DJ, Varner E, Austin JM Jr. .Treatment of the small unruptured ectopic pregnancy: a cost analysis of methotrexate versus laparoscopy. Obstet Gynecol 1996; 88(1):123-7.

15. Lipscomb GH, McCord ML, Stovall TG, Huff G, Portera SG, Ling FW. Predictors of success of methotrexate treatment in women with tubal ectopic pregnancies. N Engl J Med 1999; 341:1974-1978.

16. Stika CS,Anderson L, Frederiksen MC. Single-dose methotrexate for the treatment of ectopic pregnancy: Northwestern Memorial Hospital three-year experience. Am J Obstet Gynecol 1996; 174:1840-6.

17. Deb S; Clewes J; Hewer C; Raine-Fenning N. The management of Cesarean scar ectopic pregnancy following treatment with methotrexate--a clinical challenge. Ultrasound Obstet Gynecol 2007; 30 (6): 889-92.

18. Buster JE; Heard MJ.Current issues in medical management of ectopic pregnancy. Curr Opin Obstet Gynecol 2000; 12(6):525-7.

19. Barnhart K, Hummel AC, Sammel MD, Menon S, Jain J,Chakhtoura N. Use of "2-dose" regimen of methotrexate to treat ectopic pregnancy. Fertil Steril 2007; 87 (2):250-6.

20. Stovall T, Ling F: Ectopic pregnancy. Diagnostic and treatment algorithms minimizing surgical intervention. J Reprod Med 1992; 807-12.

21. Bueno F, Sáez J, Ocaranza M, Conejeros C, Vaccarezza I, Borquez P, et al. Tratamiento médico del embarazo ectópico. Rev Chil Obstet Gynecol 2002; 67(3): 173-9.

22. Rojas E, Hernández L, Sánchez J, Risco RJ, Pulido VH. Tratamiento médico del embarazo ectópico no roto. Ginecol Obstet Mex 2004; 72:135-41.

23. Huamán $\mathrm{H}$, Huamán $\mathrm{H}$, Arias J. Embarazo ectópico: tratamiento médico. Rev Per Ginecol Obstet 2007; 53(1):39-41. 